\title{
OC-025 IMPACT OF URGENT SUSPECTED CANCER (USC) VERSUS NON-USC REFERRAL PATHWAYS ON SURVIVAL OF UPPER GI CANCERS
}

doi:10.1136/gut.2011.239301.25

A Sugumaran, 1,* J Hurley, ${ }^{1}$ P George, ${ }^{1} \mathrm{~J}$ Pye ${ }^{2}{ }^{1}$ Gastroenterology, Wrexham Maelor Hospital, Wrexham, UK; ${ }^{2}$ Surgery, Wrexham Maelor Hospital, Wrexham, UK

Introduction Department of Health published guidance on 'Improving Outcomes in Upper Gastro-intestinal cancer' in January 2001. ${ }^{1}$ One of the important recommendations was 'Fast Track Endoscopy' service within 2 weeks for patients with suspicion of cancer ('the 2 weeks rule'). Our aim was to compare the detection of curative Upper GI cancers (Oesophagus and stomach) and survival in patients referred for Oesophago gastro duodenoscopy (OGD) via USC versus non-USC (routine) route in a District general hospital.

Methods We performed a retrospective audit on all patients referred for OGD between October 2006 and April 2007 in Wrexham maelor hospital (Cross-border Upper GI Cancer center for North Wales and West Cheshire). Data on mode of referral (USC or non-USC), endoscopic and histological diagnosis, curative or palliative intervention (if cancer detected) was collected. Patients with upper GI cancers were followed to check for survival at 18 and 36 months.

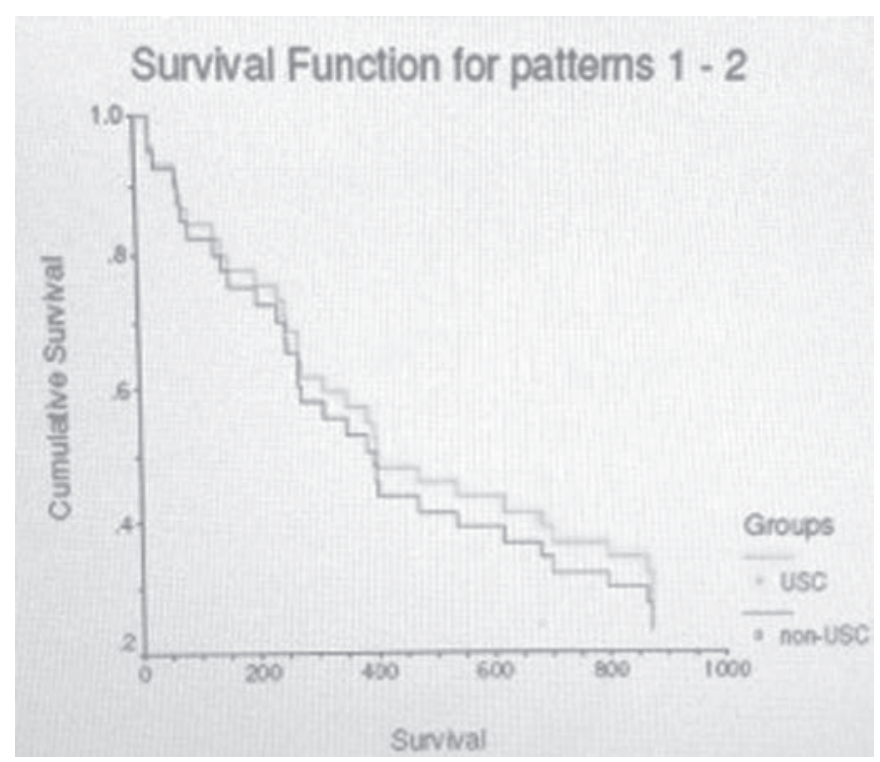

Figure 1 OC-025 Kaplan-Meier survival curve 
Results 404 patients had OGD in USC while 1538 patients via non-USC pathway. 25 Upper GI cancers were detected in USC and 18 in non-USC, out of which $28 \%$ (7) in USC and $27.7 \%$ (5) in non-USC cancers had curative intervention. Though USC pathway detected more cancers (6.2\% of all OGDs performed) than non-USC $(1.2 \%)$, there was no statistical significance in the detection of curative malignancies. At 18 months, 5 out of 7 patients who had curative treatment and 4 out of 18 with palliative approach survived in the USC group, while all 5 cured patients and 4 out of 13 palliated patients survived in the nonUSC. At 3 years, five patients in the USC who had curative treatment (and survived at 18 months) along with two of the palliated patients were alive, while in the non-USC, four cured patients survived but all palliated patients died. Cox regression analysis did not find any statistically significant survival benefit between the two groups at 18- or 36-month follow-up (Kaplan-Meier survival curve attached).

Conclusion '2-week rule' for OGD in USC patients has no statistical advantage in detection of curative upper GI malignancies nor has survival benefit when compared with the nonUSC referrals. Clarifying guidelines that would help detect 'early' cancers and resource diversion towards OGD surveillance in high-risk patients and creating public awareness are increasingly discussed as the only measures to improve survival in upper GI cancers.

Competing interests None.

Keywords 2-week-rule, endoscopy.

\section{REFERENCE}

1. http://www.dh.gov.uk/prod_consum_dh/groups/dh_digitalassets/@dh/ @en/documents/digitalasset/dh_4014513.pdf 